\title{
COMPARACIÓN DE LOS PROGRAMAS DE ACTIVIDAD FÍSICA Y DEPORTE DIRIGIDOS A LA POBLACIÓN LATINOAMERICANA
}

\author{
COMPARISON OF PROGRAMS OF PHYSICAL ACTIVITY AND \\ SPORT FOR LATIN AMERICAN POPULATION
}

\author{
Marlucio de Souza Martins ${ }^{1}$ \\ Arturo Molina Pinzón ${ }^{2}$ \\ Andrés Felipe Salazar Garcia ${ }^{3}$
}

Resumen

\begin{abstract}
Este estudio tiene como finalidad hacer un comparativo de las tendencias de políticas de Estado y de programas sociales de actividad física y deporte en los siglos Xx y xxI, y sus implicaciones en la transformación de la calidad de vida en la población latinoamericana. El análisis de los programas sociales de actividad física y deporte en las principales ciudades latinoamericanas, como Muévete Bogotá (Bogotá, D. C.), Argentina, Nuestra Cancha (Argentina), Programa Nacional de Cultura Física y Deporte (México), Actitud Activa (Ciudad de México, D. F.) y el programa Agita Mundo (São Paulo), establecen parámetros de actividad física y deporte a nivel local e internacional además de implementar estrategias para reducir las tasas de morbilidad derivadas de las enfermedades no transmisibles. Sin embargo, la ausencia de planes e intervenciones nutricionales, sumada a la falta de actividad física regular, son los principales componentes para el desarrollo de enfermedades no transmisibles, que están contribuyendo al aumento en las tasas de mortalidad. En este sentido, la Organización Mundial de la Salud - oms (2002) afirma que esos factores fueron la causa del $60 \%$ de los 56 millones de muertes anuales y del $47 \%$ de la carga mundial de morbilidad.
\end{abstract}

Palabras claves: actividad física, deporte, políticas de Estado, programas sociales, sedentarismo, enfermedades no transmisibles.

\section{Abstract}

This study aims to make a comparison of trends in public policy and social programs of physical activity and sports in the twentieth and twenty-first centuries, and its implications in transforming the quality of life in the Latin American population. The analysis of social programs of physical activity and sport in major Latin American cities, such as Muévete Bogotá (Bogotá, D. C.), Argentina, Nuestra Cancha (Argentina), Programa Nacional de Cultura Física y Deporte (México), Actitud Activa (Ciudad de México, D. F.) and the program Agita Mundo (São Paulo), establish parameters of physical activity and sports at both local and international levels and also implement strategies to reduce the rates of morbidity derived of non-communicable diseases. However, the absence of plans and nutritional strategies, coupled with the lack of regular physical activity are the main components for the development of non-communicable diseases, which are contributing to increased mortality rates. In this sense, the World Health Organization - wHO (2002) states that these factors were the cause of $60 \%$ of the 56 million annual deaths, and $47 \%$ of the global burden of morbidity.

Keywords: physical activity, sport, public policy, social programs, sedentary lifestyle, non-communicable diseases.

Fecha de recepción: 15 de julio de 2014

Fecha de aprobación: 1 de noviembre de 2014

1 Licenciado en Educación Física - Universidad Regional de Blumenau - FURB - BRASIL; Magister en Educación - Universidad Regional de Blumenau - FURB - BRASIL; Investigador del Grupo de Investigación "Universidad en Movimiento" de la Pontificia Universidad Javeriana - Bogotá; Docente del Departamento de Formación de la Pontificia Universidad Javeriana - Bogotá; Miembro del Comité del Medio Universitario de la Pontificia Universidad Javeriana- Bogotá. Correo electrónico: mdesouzamartins@javeriana.edu.co

2 Profesional de Cultura Física, Deporte y Recreación-Universidad Santo Tomás - Bogotá, D.C. - Colombia. Especialista en Ejercicio Físico para la Salud - Universidad del Rosario - Bogotá, D.C. - Colombia. Investigador del Grupo de Investigación “Universidad en Movimiento" de la Pontificia Universidad Javeriana. Docente del Departamento de Formación de la Pontificia Universidad Javeriana. Entrenador de la Condición Física - Programa Condición Física - Centro Javeriano de Formación Deportiva - Vicerrectoría del Medio Universitario - Pontificia Universidad Javeriana.. Correo electrónico: amolina@javeriana.edu.co

3 Cirujano general dedicado a la cirugía de mínima invasión en particular al manejo de la obesidad, entrenamiento en manejo multidisciplinario de la obesidad; Profesor instructor de cirugía de la Pontificia Universidad Javeriana; Especialista en Epidemiologia clínica de la Universidad del Rosario; Investigador del Grupo de Investigación “Universidad en Movimiento" de la Pontificia Universidad Javeriana. Correo electrónico: afsalazar@husi.org.co 
Para citar este artículo:

De Souza Martins, M., Molina, A., Salazar, A.F. (2014).

Comparación de los programas de actividad física y deporte

dirigidos a la población latinoamericana. Revista Lúdica

Pedagógica, (20), 79-86.

\section{PRESENTACIÓN}

La preocupación por el incremento de los niveles de sedentarismo en Latinoamérica antecede al siglo xx. Con la Revolución industrial ocurre el crecimiento de los niveles de sedentarismo y, como consecuencia, el surgimiento de enfermedades no transmisibles como: hipertensión arterial, diabetes mellitus tipo 2 y obesidad.

A partir de la Revolución industrial, la sociedad inició un proceso de modernización y globalización de nuevos estilos de vida. Se trata de un fenómeno de mutación sociocultural tecnológica conocido como "males de la sociedad moderna" con respecto a la actividad física. Vivimos actualmente en una sociedad que en el último siglo ha cambiado su modo de vida como consecuencia de la evolución tecnológica, que favorece la inactividad física y el sedentarismo. Hoy cambiamos la caminata por el uso del vehículo, cambiamos las escaleras por los ascensores; además, no podemos dejar de mencionar el sinnúmero de horas que pasamos sentados frente al computador, el televisor y las consolas de videojuegos. Blasco (1994) comparte te la misma opinión, al afirmar que la tecnología ha permitido liberar al ser humano de las tareas pesadas que requerían grandes dosis de esfuerzo físico, así como facilitarle los desplazamientos sin tener que caminar.

Los resultados de la evolución y la modernización de los estilos de vida generarán consecuencias graves para la salud y la calidad de vida de la sociedad moderna. Estos factores del "mal de la sociedad moderna" hacen que predomine la inactividad física y, como consecuencia de ella, una serie de patologías y enfermedades no transmisibles, como obesidad, atrofia ósea y muscular, alteraciones degenerativas cardiovasculares, entre otras.

Por otro lado, la practicidad y comodidad de consumir comidas rápidas traen como consecuencia directa un deterioro nutricional de la alimentación diaria, que se refleja en el aumento de los índices de colesterol y del porcentaje de grasa corporal. La ausencia de una alimentación adecuada, sumada a la falta de actividad física regular, son los principales componentes para el desarrollo de enfermedades no transmisibles, que están contribuyendo al aumento en las tasas de mortalidad y al caos en los programas de salud estatales.
En este sentido, este estudio tiene como finalidad hacer un comparativo de las tendencias de políticas de Estado y de programas sociales de actividad física y deporte en los últimos dos siglos, y sus implicaciones en la transformación de la calidad de vida en la población latinoamericana.

Se realizó un análisis de algunos programas sociales de actividad física y deporte que se han implementado en las principales ciudades latinoamericanas, como: Muévete Bogotá (Bogotá, D. C.), Argentina, Nuestra Cancha (Argentina), Programa Nacional de Cultura Física y Deporte (México), Actitud Activa (Ciudad de México, D. F.) y el programa Agita Mundo (São Paulo), que establecen parámetros de actividad física y deporte a nivel local e internacional y promulgan estrategias para reducir las tasas de morbilidad derivadas de las enfermedades no transmisibles, a partir de la adherencia a la práctica regular de actividad física y deporte, a través del desarrollo e implementación de programas sociales.

\section{PROGRAMAS SOCIALES}

La preocupante situación que constituye la inactividad física, así como los problemas y las enfermedades relacionadas con ella, constituye una tendencia mundial. Los gobiernos de diferentes naciones llevan varios años implementando medidas y estrategias de trabajo en conjunto con las empresas tanto públicas como privadas y la comunidad, con el fin de revertir las cifras publicadas por la Organización Mundial de la Salud - OMS (2010) en las últimas décadas con respecto al impacto del sedentarismo.

La idea principal de un programa social para combatir dicho problema está en la creación o generación de políticas de largo plazo que trasciendan los gobiernos y que generen un entorno favorable para que las personas puedan mejorar sus hábitos de vida saludable. Además, se debe plantear como objetivo bajar los altos costos para los hospitales y sistemas públicos de salud destinados a la atención y el seguimiento de personas con enfermedades no transmisibles derivadas del sedentarismo.

En el caso de Latinoamérica (Argentina, Brasil, Colombia y México) los estudios demuestran que más de un $50 \%$ de la población es irregularmente activa; en el panorama colombiano, el índice de inactividad física llega al 79\% (Matsudo y Matsudo, 2005). Los gobiernos vienen desarrollando acciones de promoción y prescripción de la actividad física en relación con la salud 
para la prevención de enfermedades no transmisibles con la intención de disminuir los índices de sedentarismo. En este sentido, los principales programas de actividad física y deporte de Latinoamérica tienen como propósito común reducir la prevalencia de la inactividad física en la población general, promoviendo la adopción de estilos de vida activos.

Para visualizar el panorama de los programas de actividad física y deporte en Latinoamérica, a continuación presentamos las síntesis centrales de algunos de los programas implementados en las principales ciudades de Argentina, Brasil, Colombia y México.

Panorama en Argentina

El Programa Nacional de Actividad Física para la Salud "Argentina Activa y Saludable" fue implementado a partir de los resultados estadísticos publicados en 2000 por el Instituto Nacional de Estadística y Censos - Indec (2000), los cuales demostraron que el $40 \%$ de los argentinos tienen sobrepeso y que el $60 \%$ de la población es sedentaria. Por esta razón, el Gobierno inició una campaña nacional que busca reducir los altos índices de sedentarismo promoviendo una cultura que revalorice el cuidado del cuerpo y la salud.

A partir del año 2003, el Gobierno Nacional argentino inició el programa Argentina, Nuestra Cancha en asocio con instituciones de educación superior y universidades para contribuir activamente con las políticas de Estado. El programa propone la creación de polos de actividad física y deportiva totalmente gratuitos para todas las edades, en todo el territorio nacional.

Conforme al Ministerio de Desarrollo Social (2013), el objetivo del programa es reducir el sedentarismo promoviendo una cultura que revalorice el cuidado del cuerpo, la salud y la calidad de vida, además de involucrar a la población general en actividades que puedan disminuir los problemas derivados del sedentarismo, como enfermedades no transmisibles, además de los problemas producidos por el tabaquismo y el alcoholismo.

Panorama en Brasil

Actualmente la inactividad física en Brasil sobrepasa el $65 \%$ de la población nacional. En el departamento de São Paulo, los datos estadísticos son más preocupantes debido a la alta prevalencia de estilos de vida sedentarios, que supera el $60 \%$ en hombres y $80 \%$ en mujeres (Matsudo y Matsudo, 2005). Con este panorama surgió la necesidad de implementar intervenciones para pro- ducir un cambio en la conducta de la población y reducir la prevalencia de estilos de vida sedentarios.

En 1995, inició el programa Agita São Paulo con el objetivo de fomentar la actividad física para combatir los problemas generados por el sedentarismo y promover un mecanismo de promoción de la salud, con el enfoque de disminuir los costos de los planes de salud en la salud pública nacional. El programa Agita São Paulo fue el pionero en Latinoamérica al implementar programas sociales de intervención de estilos de vida. El país ha completado más de dos décadas contribuyendo en el campo del deporte, de la aptitud física y la promoción de la actividad física en observancia a los cambios de estilos de vida.

Los investigadores han encontrado que adquirir un hábito saludable trae consigo la adopción de otros, que ayudan a disminuir la incidencia de enfermedades como la obesidad y la diabetes. Con respecto al impacto que pueden tener estas políticas, se calcula que la disminución del sedentarismo en São Paulo del 9.6\% al 2.7\% entre el 2002 y el 2008 generó un ahorro al sistema de salud de dicho estado de aproximadamente 310 millones de dólares al año.

Con el impacto positivo del programa Agita São Paulo, a partir de 1998 han comenzado a surgir iniciativas similares en otros países, para fomentar un impulso internacional de un estilo de vida más activo como un elemento crucial del bienestar y de la salud física y mental de la población. En 2002 surgió el programa Agita Mundo, que ha promovido la investigación y la diseminación de información acerca de los beneficios de la actividad física y de las estrategias para aumentarla, como también ha apoyado la creación de programas y redes nacionales e internacionales para promover la actividad física.

\section{Panorama en Colombia}

Según la oms (2002), Colombia es el sexto país latinoamericano al que más se le atribuyen muertes por inactividad física. Estudios realizados por Vidarte et ál. (2012) determinarán que el nivel de sedentarismo tiene una prevalencia del $73 \%$ en la población general colombiana; por género, el nivel de sedentarismo en hombres asciende al $61 \%$ y en mujeres al $84 \%$. Como resultado de este tipo de conducta sedentario, en Colombia en el año 2008 se presentaron 109.405 muertes por enfermedades del aparato circulatorio, enfermedad isquémica del corazón, enfermedades cerebrovasculares y diabetes mellitus en personas mayores de 45 años (Garzón y Rodríguez, 2012). 
El Gobierno Nacional de Colombia promulgó en el año 2009 la Ley 1355, en la cual se definen la obesidad y las enfermedades crónicas no transmisibles como una prioridad de salud pública, y se adoptan medidas para su control, atención y prevención. En el artículo 5 se establecen las estrategias para promover la actividad física y se conmina al Ministerio de Educación Nacional $\mathrm{y}$ a las instituciones educativas para que promuevan el incremento y la calidad de las clases de Educación Física con personal idóneo y adecuadamente formado. A su vez, se estableció, en cabeza del Ministerio de la Protección Social, el compromiso de reglamentar mecanismos para que todas las empresas del país promuevan durante la jornada laboral pausas activas para todos sus empleados. Por otro lado, el artículo 6 habla sobre la promoción del transporte activo como estrategia de prevención de la obesidad.

La estrategia para cambiar este cuadro de sedentarismo ha generado que el Gobierno colombiano, por medio de sus departamentos, haya elaborado programas de actividad física gratuita realizados en plazas, parques y centros comunitarios. El programa más conocido es Muévete Bogotá, que cumple quince años de actividad y realiza diferentes actividades masivas, que buscan promover la práctica de actividad física, para que la población pueda tener una vida saludable.

El programa Muévete Bogotá, iniciado en el año de1998, es una estrategia de intervención que busca modificar el estilo de vida de la población por medio de la inclusión de la actividad física con el fin de contribuir con la formación integral de los ciudadanos y de mejorar la calidad y expectativa de vida.

Como resultado de este tipo de políticas, en el año 2009 en Bogotá se elaboró un programa de deporte, recreación y actividad física proyectado a diez años (20092019), llamado Bogotá más Activa, el cual centra sus esfuerzos en mejorar las condiciones ambientales e institucionales que permitan incrementar los niveles de práctica del deporte, la recreación y la actividad física con el fin de aportar beneficios a la salud física, emocional y psíquica de las personas.

\section{Panorama en México}

Entre las diez causas principales identificadas por la oMs como los factores más peligrosos para la salud relacionados con el estilo de vida está el sedentarismo. Los datos de inactividad física en México son verdaderamente alarmantes, según el estudio del Centro de Estudios Sociales - México (2012), el sedentarismo se evidencia con mayor preponderancia en el $80 \%$ de las mujeres y en el $63 \%$ de los hombres. De acuerdo con la Encuesta Nacional de Salud y Nutrición del Instituto Nacional de Salud Pública - INSP (2010), en el país tres de cada diez personas tienen obesidad y siete de cada diez personas presentan sobrepeso. El Gobierno mexicano considera el sedentarismo como uno de los factores de riesgo de mortalidad más importantes en el país.

Como parte de la política social del Gobierno mexicano, el Programa Nacional de Cultura Física y Deporte (2013) establece los objetivos, las estrategias y las líneas de acción para lograr incorporar, en la población en general, la práctica de la actividad física y deportiva como un medio para mejorar su calidad de vida y fomentar una cultura de la salud. En su plan de desarrollo 2013-2018, establece promover acciones de protección, promoción y prevención para el mejoramiento de la salud por medio del deporte social. El Ministerio de Educación, Cultura y Deporte (2014) define el:

Deporte social como actividades deportivas que promueven, fomentan y estimulan el que todas las personas sin distinción de género, edad, discapacidad, condición social, religión, opiniones, preferencias o estado civil, tengan igualdad de participación en actividades deportivas con finalidades recreativas, educativas y de salud o rehabilitación. (Ministerio de Educación, Cultura y Deporte, 2014, p. 21)

En este sentido, el Gobierno mexicano promueve el deporte social por medio de los gimnasios urbanos, gratuitos instalados en parques públicos; convenios con Clubes deportivos sociales para desarrollar actividades deportivas y recreativas, fomentando la integración social. A su vez los programas Actitud Activa tienen por objetivo fomentar un estilo de vida activo y saludable por medio del ejercicio físico; están dirigidos a los trabajadores y sus familias. El programa Ponte al 100 tiene como objetivo contribuir a disminuir los índices de sobrepeso y obesidad por medio de mediciones, evaluaciones y prescripción de ejercicios y dietas personalizadas.

\section{LINEAMIENTOS DE ACTIVIDAD FÍSICA Y SALUD}

Al analizar los programas sociales de actividad física y deporte en las principales ciudades latinoamericanas, se encuentra un objetivo común, que es el de vencer en la lucha contra el sedentarismo, a la vez que se establecen parámetros de actividad física y deporte 
a nivel local e internacional y se promulgan estrategias para reducir las tasas de morbilidad derivadas de las enfermedades no transmisibles. Las estrategias de prevención y control de las enfermedades crónicas no transmisibles adoptadas por las principales ciudades latinoamericanas manejan principalmente tres niveles de acción: desarrollo de políticas, actividades comunitarias y servicios de atención en salud.

Lamentablemente, los programas sociales implementados en Latinoamérica, así como los esfuerzos para incorporar la prevención, fomentar hábitos en actividad física y controlar el impacto de enfermedades crónicas no transmisibles no presentan un resultado efectivo en disminuir el crecimiento del sedentarismo en Latinoamérica.

La inactividad física es un factor predictor importante de enfermedad cardiovascular, diabetes mellitus tipo 2, obesidad, algunos cánceres, salud deficiente del sistema óseo, algunos aspectos de la salud mental y mortalidad global, así como una precaria calidad de vida (Hallal, 2012).

Los cambios hacia estilos de vida más sedentarios y alimentación poco saludable están teniendo un gran impacto en Latinoamérica. Según la oms (2002) los factores derivados del sedentarismo fueron la causa del $60 \%$ de los 56 millones de muertes anuales y del $47 \%$ de la carga mundial de morbilidad. Se estima que las enfermedades crónicas son responsables del $60 \%$ de todas las muertes en 2005 y que el $80 \%$ ocurren en países de ingresos bajos y medianos (OMS, 2005). Según la oms (2007), el sedentarismo se constituye como una amenaza creciente por fomentar hábitos como una alimentación poco sana y el consumo de tabaco. Esto significa que si no ocurre un cambio en los estilos de vida hacia unos más saludables, dentro de los próximos diez años los problemas relacionados con enfermedades no transmisibles aumentarán en un $17 \%$, alcanzando los 64 millones de personas que fallecerán por consecuencia del sedentarismo.

Según el Conjunto de Acciones para la Reducción Multifactorial de Enfermedades No Transmisibles - Carmen (2003), los factores de riesgo más importantes de enfermedades crónicas incluyen: hipertensión arterial, alto índice de colesterol (superior a $200 \mathrm{mg}^{*} \mathrm{dL}^{-1}$ ), ingesta inadecuada de frutas y verduras, sobrepeso y obesidad, inactividad física y consumo de tabaco. Cinco de estos factores están relacionados con la actividad física y el régimen alimentario. En total, los grandes factores de riesgo son responsables aproximadamente del $80 \%$ de las muertes por enfermedades cardiovasculares y ataques cardiacos fulminantes.

En este sentido, para frenar y prevenir el crecimiento de las enfermedades no transmisibles que amenazan la salud en Latinoamérica, la oms (2008) propone que las políticas y planes nacionales sobre actividad física deben abarcar múltiples estrategias orientadas al apoyo de la población y crear un ambiente solidario. También sugiere incluir en los programas sociales:

Campañas comunitarias usando medios de comunicación de masas para elevar la conciencia sobre la importancia y los beneficios de la actividad física en la población entera y diseminar mensajes que la promuevan; acceso mejorado a lugares para el desarrollo de la actividad física; provisión de escenarios de recreo de fácil acceso para niños; construcción de caminos; estrategias para el transporte activo al trabajo (ir en bicicleta y caminar); consejos de cuidado y bienestar principalmente orientados a la población de adultos mayores; formación de redes sociales que promuevan la actividad física. (OMS, 2008, p. 5)

Estudios recientes muestran cómo la disminución de la actividad física en las últimas cinco décadas explica un descenso en el gasto energético del individuo, lo que redunda en gran parte en el aumento de peso de la población, tanto al disminuir la cantidad de energía utilizada en actividades laborales (Church, 2011) como en las actividades del hogar (Archer, 2013).

La inactividad física y el cigarrillo son los dos factores de riesgo más importantes para enfermedades crónicas no transmisibles, y cada uno de estos se asocia a 5 de los 36 millones de muertes anuales por esta causa (WHo, 2009). Sin embargo, los esfuerzos para combatir la inactividad física han sido mucho menores y de más lenta implementación que los que se han realizado con otros factores de riesgo, como tabaco, dieta y alcohol, y su impacto en la sociedad aún no ha sido significativo (Das, 2012).

Es interesante examinar el problema también desde la perspectiva de la ausencia de planes y estrategias nutricionales. Sumado a la falta de actividad física regular, este es uno delos principales componentes para el desarrollo de enfermedades no transmisibles, que están contribuyendo al aumento en las tasas de mortalidad. En este sentido, la oms (2007) como parte de la estrategia mundial sobre el régimen alimentario, la actividad física y la salud organizó un foro con el objetivo de formular 
recomendaciones e intervenciones encaminadas a reducir el consumo de sal en toda la población, con la meta a largo plazo de prevenir enfermedades no transmisibles.

De acuerdo con la oms (2007) "la ingesta alimentaria de sodio procedente de todas las fuentes influye en los niveles de presión arterial de la población y debe limitarse para reducir el riesgo de padecer cardiopatías coronarias y accidentes cerebrovasculares" (p. 3). En este sentido, la estrategia para combatir las enfermedades no transmisibles aborda dos principales factores: el régimen alimentario y la actividad física.

$\mathrm{Al}$ analizar las estrategias de prevención y control de las enfermedades crónicas no transmisibles adoptadas por las principales ciudades latinoamericanas, se contrasta, de manera general, que pocos países tienen una política nacional que promueva medidas sostenibles para la reducción de las tasas de morbilidad y de mortalidad relacionadas con intervención conjunta de hábitos alimentarios y actividad física. De acuerdo con la oms (2007): "en América del Sur, unos pocos países que han desarrollado recomendaciones generales ('reducción del consumo de sal', 'moderación en el consumo de sal'), y Brasil es el único país con una recomendación nutricional (<5 g/d de sal)" (p. 16).

El 72\% de la sal consumida a diario se encuentra en alimentos procesados, especialmente en embutidos, pan y panes especiales, quesos y platos preparados (comidas rápidas); también se calcula que aproximadamente el $70 \%$ de la sal consumida procede de alimentos consumidos fuera del hogar. En comparación con los países europeos, la mayoría cuentan con una amplia gama de recomendaciones cuantitativas y/o cualitativas sobre el consumo de sal, como también se han ejecutado medidas específicas para reducir el consumo de sal en toda la población como parte de una política nutricional nacional conjuntamente con políticas de actividad física y deporte.

Según la oms (2013):

Disminuir la ingesta de sodio reduce de forma significativa la tensión arterial sistólica y diastólica en los adultos y niños [...] un mayor consumo de sodio se asocia con un riesgo más alto de accidentes cerebrovasculares [...] así como los posibles efectos adversos en la lipemia, las concentraciones de catecolaminas y la función renal. (p. 4)

A partir de los cálculos de la oms (2009), se establece que la hipertensión sistólica es una causa de muerte por enfermedades cardiovasculares, responsable del $51 \%$ de los accidentes vasculares cerebrales y del $45 \%$ de las muertes por enfermedades cardiacas isquémicas.

A pesar de que el sodio es necesario para el correcto funcionamiento del organismo, la sal consumida en exceso tiene varias consecuencias. De acuerdo con la oms (2010), además de los riesgos de enfermedades cardiovasculares, el consumo de sodio en exceso produce consecuencias no sistémicas, como: dificulta la función de los riñones; disminuye la cantidad de calcio; ocurre la retención de líquidos; favorece el desarrollo de algunos tipos de tumores gástricos; dificulta la función del aparato respiratorio y predispone al sobrepeso y la obesidad.

Por otro lado, la obesidad es una epidemia de proporciones inmensas, teniendo en cuenta que afecta hasta un $66.3 \%$ de la población estadounidense (Ogden, 2006), y representa uno de los mayores factores de riesgo para enfermedades crónicas no transmisibles, tales como enfermedad cardiovascular, enfermedad coronaria, falla cardiaca, eventos cerebrovasculares, disfunción ventricular y arritmias (Klein, 2004).

Se ha demostrado que una pérdida de peso modesta presenta un beneficio para el impacto de estas enfermedades en el organismo; la actividad física y el entrenamiento con ejercicio son parte fundamental para lograrla (Swift, 2013). Donelly (2009) y Haskell (2007) recomiendan el ejercicio como parte fundamental para mantener y mejorar la salud, para prevenir la ganancia de peso, para promover la pérdida de peso clínicamente significativa y para prevenir la re-ganancia de peso posterior a la pérdida significativa de peso.

\section{CONCLUSIÓN}

Se ha comprobado que los programas sociales de actividad física y deporte en las principales ciudades latinoamericanas establecen parámetros de actividad física y deporte a nivel local e internacional que incluyen simultáneamente acciones por parte de las instituciones y grupos de interés de los sectores públicos y privados, con un objetivo en común: combatir los estilos de vida sedentarios.

Los programas sociales de actividad física y deporte son de gran importancia para la promoción de un estilo de vida más activo y para la prevención de las enfermedades no transmisibles en la población latinoamericana. Fomentar la práctica de una actividad física regular por medio de programas sociales genera un entorno favora- 
ble para que las personas puedan mejorar sus hábitos de vida saludable. La práctica diaria de 30 minutos de actividad física reduce el riesgo cardiaco, disminuye la presión arterial, regula el colesterol, mejora la calcificación de los huesos, aumenta la masa muscular, reduce el sobrepeso y disminuye la incidencia de diabetes y accidentes vasculares cerebrales.

Las políticas públicas de actividad física tienen que estar enmarcadas dentro de programas sociales que abarquen el desarrollo integral desde los componentes físicos, el desarrollo nutricional y social que serán ejes multidisciplinares que contribuirán para el éxito en la disminución del panorama de enfermedades no transmisibles y otras enfermedades crónicas presentadas en la población latinoamericana.

Cabe resaltar la importancia de la orientación de los programas de educación física en los niveles de escolaridad desde el preescolar hasta el universitario, como también los programas de Estado, para responder a las necesidades poblacionales identificadas a partir de estudios de hábitos y estilos de vida sedentarios, que serán el punto de partida para determinar las estrategias de intervención en actividad física, nutrición y promoción de la salud.

\section{REFERENCIAS BIBLIOGRÁFICAS}

Archer, E., Shook, R.P., Thomas, D. M. et ál. (2013). 45-Year trends in women's use of time and household management energy expenditure. PLOS ONE.

Blasco, T. (1994). Actividad física y salud. Martínez Roca, Barcelona.

Carmen (2003). Conjunto de acciones para la reducción multifactorial de enfermedades no transmisibles. Organización Panamericana de la Salud. Washington: EUA.

Church, T.; Thomas, D.; Tudor-locke, C., et ál. (2011). Trends over 5 decades in U.S. occupation-related physical activity and their associations with obesity. PLOS ONE.

Das P., Horton, R. (2012). Rethinking our approach to physical activity. Lancet.

Donnelly, J.; Blair, S.; Jakicic, J. et ál. (2009). Appropriate physical activity intervention strategies for weight loss and prevention of weight regain for adults. Med Sci Sports Exerc. 41(2):459-471. doi: 10.1249/ MSS.0b013e3181949333.
Indec (2000). Indicadores básicos: Argentina 2000. Ministerio de la Salud Argentina.

Garzón, N. \& Rodríguez, D. (2012). Desarrollo de intervenciones en actividad física en Brasil y Colombia. Bogotá: Universidad Nacional de Colombia.

Hallal, P.; Bauman, A.; Heath, G., et ál. (2012). Physical activity: more of the same is not enough. Lancet.

Haskell, W.; Lee, I.; Pate, R., et ál. (2007). Physical activity and public health: updated recommendation for adults from the American College of Sports Medicine and the American Heart Association. Med Sci Sports Exerc. 39(8):1423-34.

Klein, S., Burke, L., Bray, G., et ál. (2004).Clinical implications of obesity with specific focus on cardiovascular disease: a statement for professionals from the American Heart Association Council on Nutrition, Physical Activity, and Metabolism: Endorsed by the American College of Cardiology Foundation. Circulation.

Matsudo, S. y Matsudo, V. (2005) Agita São Paulo: Fomento de la actividad física como modo de vida en Brasil. São Paulo.

Ministerio de Desarrollo Social (2013). Plan Nacional de Deporte Social (2013-2016): Programa Nacional de Actividad Física para la Salud. Gobierno Nacional Argentino.

Ministerio de Educación, Cultura y Deporte (2014). Programa Nacional de Cultura Física y Deporte 20142018. México: Gobierno Mexicano.

Ogden, C.L., Carroll, M.D., Curtin, L.R., et ál. (2006). Prevalence of overweight and obesity in the United States, 1999-2004. JAMA.

oms (2002).The world health report 2002. Reducing risks, promoting healthy life. Geneva, Switzerland: World Health Organization.

oms (2005). Preventing chronic diseases: a vital investment. Geneva, Switzerland: World Health Organization.

oms (2007). Informe de un foro y reunión técnica de la oms del 5 al 7 de octubre del 2006, Paris. Geneva, Switzerland: World Health Organization.

oms (2008). Una guía de enfoques basados en población para incrementar los niveles de actividad física: aplicación de la estrategia mundial sobre régimen alimentario, actividad física y salud.Geneva, Switzerland: World Health Organization. 
oms (2009). Global Health Risks: Mortality and burden of disease attributable to selected major risk. Geneva, Switzerland: World Health Organization.

oms (2010). Creating and enabling environment for population-based salt reduction strategies. Report of a joint technical held by WHO and the Food Standards Agency, United Kingdom, July 2010. Geneva, Switzerland: World Health Organization.

o MS (2013). Directrices: ingesta de sodio en adultos y niños. Geneva, Switzerland: World Health Organization.
Swift, D. L., Lavie, C. J., Johannsen, N.et ál. (2013). Physical activity, cardiorespiratory fitness, and exercise training in primary and secondary coronary prevention.

Vidarte-Claros, J., Vélez, C. y Parra, J.H. (2012). Niveles de sedentarismo en población de 18 a 60 años. Rev. Salud Pública, 14 (3), 417-428.

WHO (2009).Global health risks: mortality and burden of disease attributable to selected major risks. Geneva, World Health Organization. 